\title{
There Are No Classical Glueballs ${ }^{\star}$
}

\author{
Sidney Coleman \\ Lyman Laboratory of Physics, Harvard University, Cambridge, Massachusetts 02138, USA
}

\begin{abstract}
I show that there are no finite-energy non-singular solutions of classical Yang-Mills theory in four-dimensional Minkowski space that do not radiate energy out to spatial infinity. Finite-energy non-singular solutions that are either static or periodic in time, are a fortiori non-radiant; thus this generalizes earlier theorems that state that there are no such solutions.
\end{abstract}

\section{Introduction}

In a linear classical field theory, like free electrodynamics, any non-singular initial configuration of fields of finite energy will eventually spread out over all space; whatever the initial configuration, the final state is simply outgoing radiation. In contrast, certain non-linear field theories are known to have finite-energy nonsingular solutions that can be described as lumps of energy held together by their own self-interaction; no energy is radiated to spatial infinity [1]. For brevity, I will call such solutions "lumps". I emphasize that lumps, as I define them, may have arbitrary time-dependence, as long as they do not radiate away any of their energy.

In this note I show that classical Yang-Mills theory in four-dimensional Minkowski space has no lumps. This extends the known theorems that state that this theory possesses no finite-energy non-singular solutions that are either timeindependent $[1,2]$ or periodic in time [3].

If Yang-Mills lumps had existed, they would have been classical analogues of the "glueballs" of quantum chromodynamics, colorless bound states composed exclusively of gauge-field quanta ("gluons"). Of course, the nonexistence of classical glueballs says nothing against the existence of quantum glueballs, anymore than the instability of the classical Hydrogen atom says anything against the existence of the quantum Hydrogen atom. However, it does indicate that it is unlikely that insight into the structure of quantum glueballs will be obtained by studying the classical limit.

* Research supported in part by the National Science Foundation under Grant No. PHY75-20427 
Section 2 is the argument against classical glueballs, with the proof of a lemma omitted. Section 3 proves the lemma.

\section{The Argument against Classical Glueballs}

I will begin by summarizing some familiar equations. The dynamical variables of Yang-Mills theory are a set of vector fields $A_{\mu}^{a}$, where $\mu$ is a space-time index ${ }^{1}$ and $a$ is an internal index. The field strength, $F_{\mu \nu}^{a}$, are defined by

$$
F_{\mu \nu}^{a}=\partial_{\mu} A_{\nu}^{a}-\partial_{\nu} A_{\mu}^{a}+c^{a b c} A_{\mu}^{b} A_{\nu}^{c},
$$

where the $c$ 's are the structure constants of a compact Lie group. The equations of motion are

$$
\partial^{\mu} F_{\mu \nu}^{a}+c^{a b c} A^{\mu b} F_{\mu \nu}^{c}=0 \text {. }
$$

The energy-momentum tensor is

$$
\theta^{\mu \nu}=F^{\mu \gamma a} F_{\gamma}^{v a}-\frac{1}{4} g^{\mu \nu} F_{\gamma \sigma}^{a} F^{a \sigma \gamma} .
$$

This obeys

$$
\partial_{\mu} \theta^{\mu v}=0,
$$

and

$$
\theta_{\mu}^{\mu}=0 \text {. }
$$
fields,

It is sometimes convenient to define the analogues of electric and magnetic

$$
E_{i}^{a}=F_{i 0}^{a}, \quad H_{i}^{a}=\frac{1}{2} \varepsilon_{i j k} F_{j k}^{a} .
$$

In terms of these,

$$
\theta^{00}=\frac{1}{2}\left(\boldsymbol{E}^{a} \cdot \boldsymbol{E}^{a}+\boldsymbol{H}^{a} \cdot \boldsymbol{H}^{a}\right),
$$

and

$$
\theta^{0 i}=\left(\boldsymbol{E}^{a} \times \boldsymbol{H}^{a}\right)^{i} .
$$

The center of energy of any non-singular solution of the field equations moves with velocity $\boldsymbol{P} / E$, where $\boldsymbol{P}$ is the momentum, the space integral of $\theta^{0 i}$, and $E$ is the energy, the space integral of $\theta^{00}$. From Equations (7) and (8), for any unit vector, $\boldsymbol{e}$,

$$
\left|e_{i} \theta^{0 i}\right| \leqq \theta^{00} \text {. }
$$

Thus, $|\boldsymbol{P}| \leqq E$. One can do better than this; in Section 3 I shall prove the following:

Lemma. The only finite-energy non-singular solution of the Yang-Mills equations for which $|\boldsymbol{P}|=E$ is the vacuum solution, $F_{\mu \nu}^{a}=0$.

Thus lumps, if they exist, must travel at less than the speed of light, and, by a Lorentz transformation, we can reduce the case of a general lump to that of a lump

Greek indices range from 0 to 3 : latin indices from the beginning of the alphabet range from 1 to the dimension of the Lie algebra : latin indices from the middle of the alphabet range from 1 to 3 . Summation over repeated indices is implied. The signature of the metric tensor is $(+---)$ 
at rest. At large distance from the center of energy of the lump, the field strengths must go to zero. Therefore, the fields far from the lump must obey the linearized field equations; that is to say, they must be the sum of a Coulomb field, an incoming radiation field, and an outgoing radiation field. By assumption, the outgoing radiation field vanishes. Hence, for any positive number $\varepsilon$ less than $1 / 2$,

$$
\lim _{|x| \rightarrow \infty}|x|^{3 / 2+\varepsilon} F_{\mu \nu}^{a}(x, t)=0
$$

uniformly in direction and uniformly in $t$, for positive $t$.

I stress that it is the uniformity in $t$ of the limit that tells us that there is no outgoing radiation. For example, for an expanding shell of radiation of finite thickness, Equation (10) would be valid for any $t$, but the limit would not be uniform in $t$; for later and later times, we would have to go to greater distances to get past the shell.

I shall now prove that the only non-singular solution of the Yang-Mills equation for which the limit of Equation (10) is uniform in $t$ for positive $t$ is the vacuum solution ${ }^{2}$.

Let us define

$$
F(r, t)=-\int_{|x| \leqq r} d^{3} x x_{i} \theta^{0 i}
$$

By Equations (4) and (5),

$$
\partial_{0} F(r, t)=\int_{|\boldsymbol{x}| \leqq r} d^{3} x 0^{00}+\int_{|\boldsymbol{x}|=\boldsymbol{r}} d^{2} S_{i} x_{j} \theta^{i j}
$$

Where $d^{2} S_{i}$ is the outwardly-directed vector element of surface area.

By assumption, as $r$ goes to infinity, the right-hand side of Equation (12) goes to $E$, uniformly in $t$ for positive $t$. Thus, in particular, there exists an $r$ such that

$$
\partial_{0} F(r, t) \geqq E / 2
$$

for all positive $t$. Hence, for this $r$,

$$
F(r, t) \geqq E t / 2+F(r, 0)
$$

for all positive $t$.

On the other hand, by Equation (9),

$$
|F(r, t)| \leqq r \int_{|x| \leqq r} d^{3} x \theta^{00} \leqq r E .
$$

The only way in which the inequalities (14) and (15) can be consistent for all positive $t$ is if $E$ vanishes. This completes the argument.

\section{Proof of the Lemma}

We wish to study non-singular solutions of the Yang-Mills equations for which $|\boldsymbol{P}|=E$. Let us choose $\boldsymbol{P}$ to be parallel to the 3-axis. Then, by Equation (9),

$$
\theta^{0 i}=\delta^{i 3} \theta^{00}
$$

2 This proof is basically a refinement of Pagel's exploitation, in Ref. [3], of the scale invariance of the theory [Eq. (5)] 
This implies

$$
\boldsymbol{H}^{a}=\boldsymbol{k} \times \boldsymbol{E}^{a}, \quad \boldsymbol{E}^{a}=-\boldsymbol{k} \times \boldsymbol{H}^{a},
$$

where $\boldsymbol{k}$ is the unit vector in the 3-direction. If we introduce the standard light-cone variables,

$$
x^{ \pm}=x^{0} \pm x^{3}
$$

Equation (17) can be recast as

$$
F_{-\mu}^{a}=0,
$$

and

$$
F_{12}^{a}=0 \text {. }
$$

Equation (20) tells us that we can always choose a gauge such that

$$
A_{1}^{a}=A_{2}^{a}=0 \text {. }
$$

This still leaves us the freedom to make a gauge transformation that depends only on $x^{+}$and $x^{-}$. In a gauge obeying Equation (21),

$$
F_{-1}^{a}=-\partial_{1} A_{-}^{a}, \quad F_{-2}^{a}=-\partial_{2} A_{-}^{a} .
$$

Thus, Equation (19) tells us that $A_{-}^{a}$ is independent of $x^{1}$ and $x^{2}$, and we can use our remaining gauge freedom to gauge it away:

$$
A_{-}^{a}=0 \text {. }
$$

This still leaves us the freedom to make a gauge transformation that depends only on $x^{+}$.

All that remains is $A_{+}^{a}$. We now invoke the + component of the field equations. By Equations (19) and (21), most of the terms in Equation (2) vanish, and we are left with

$$
\left(\partial^{1} \partial_{1}+\partial^{2} \partial_{2}\right) A_{+}^{a}=0 .
$$

The only non-singular solutions of this consistent with finiteness of the energy are those for which $A_{+}^{a}$ is independent of $x^{1}$ and $x^{2}$. By the vanishing of $F_{+-}^{a}, A_{+}^{a}$ is also independent of $x^{-}$. Thus, $A_{+}^{a}$ depends only on $x^{+}$, and we can use our last bit of remaining gauge freedom to gauge it away. This completes the proof.

Acknowledgement. I thank Edward Witten for conversations.

\section{References}

1. For a review, see Coleman,S.: Classical lumps and their quantum descendants. In: New phenomena in subnuclear physics. New York and London: Plenum Publishing Co. (to be published)

2. Deser,S.: Phys. Letters 64B, 463 (1976)

3. Pagels,H.: Rockefeller University Report C00-2232B-1236 (1977)

Communicated by A. Jaffe

Received May 2, 1977 